\title{
Induction of autophagy restores the loss of sevoflurane cardiac preconditioning seen with prolonged ischemic insult.
}

\author{
Mayumi Shiomi \\ Osaka Medical College \\ Masami Miyamae \\ Osaka Dental University
}

Genzou Takemura

Gifu University Graduate School of Medicine

Kazuhiro Kaneda

Osaka Dental University

F8IFohitakki lnarmuląational works at: https://jdc.jefferson.edu/cardiologyfp Ocaka Dental University

Part of the Cardiology Commons

\section{Let us know how access to this document benefits you See next page for additional authors}

\section{Recommended Citation}

Shiomi, Mayumi; Miyamae, Masami; Takemura, Genzou; Kaneda, Kazuhiro; Inamura, Yoshitaka; Onishi, Anna; Koshinuma, Shizuka; Momota, Yoshihiro; Minami, Toshiaki; and Figueredo, M.D., Vincent M., "Induction of autophagy restores the loss of sevoflurane cardiac preconditioning seen with prolonged ischemic insult." (2014). Division of Cardiology Faculty Papers. Paper 58. https://jdc.jefferson.edu/cardiologyfp/58

This Article is brought to you for free and open access by the Jefferson Digital Commons. The Jefferson Digital Commons is a service of Thomas Jefferson University's Center for Teaching and Learning (CTL). The Commons is a showcase for Jefferson books and journals, peer-reviewed scholarly publications, unique historical collections from the University archives, and teaching tools. The Jefferson Digital Commons allows researchers and interested readers anywhere in the world to learn about and keep up to date with Jefferson scholarship. This article has been accepted for inclusion in Division of Cardiology Faculty Papers by an authorized administrator of the Jefferson Digital Commons. For more information, please contact: JeffersonDigitalCommons@jefferson.edu. 


\section{Authors}

Mayumi Shiomi; Masami Miyamae; Genzou Takemura; Kazuhiro Kaneda; Yoshitaka Inamura; Anna Onishi; Shizuka Koshinuma; Yoshihiro Momota; Toshiaki Minami; and Vincent M. Figueredo, M.D. 


\title{
Induction of Autophagy Restores the Loss of Sevoflurane Cardiac Preconditioning Seen with Prolonged Ischemic Insult
}

\author{
Mayumi Shiomi*, Masami Miyamae ${ }^{\dagger}$, Genzou Takemura ${ }^{\circledR}$, Kazuhiro Kaneda ${ }^{\#}$, Yoshitaka \\ Inamura $^{\#}$, Anna Onishi”, Shizuka Koshinuma ${ }^{\#}$, Yoshihiro Momota ${ }^{\#}$, Toshiaki Minami*, \\ Vincent M. Figueredoll
}

*Department of Anesthesiology, Osaka Medical College, Osaka, Japan

${ }^{\dagger}$ Departments of Internal Medicine and "Anesthesiology, Osaka Dental University, Osaka, Japan, ${ }^{\&}$ Department of Cardiology, Gifu University Graduate School of Medicine, Gifu, Japan and IInstitute for Heart and Vascular Health, Einstein Medical Center, and Jefferson Medical College, Philadelphia, USA

Corresponding Author \& Reprints: Masami Miyamae, MD, PhD

Department of Internal Medicine,

Osaka Dental University

8-1 Kuzuha hanazono-cho Hirakata,

Osaka 573-1121, Japan

TEL: 81-72-864-3079, FAX: 81-72-864-3179

E-mail: miyamae0907@gmail.com

Short running title: Sevoflurane preconditioning and autophagy 


\begin{abstract}
Background: Sevoflurane preconditioning (SPC) against myocardial ischemia-reperfusion injury is lost if the ischemic insult is too long. Emerging evidence suggests that induction of autophagy may also confer cardioprotection against ischemia-reperfusion injury. We examined whether induction of autophagy prolongs SPC protection during a longer ischemic insult.
\end{abstract}

Methods: Isolated guinea pigs hearts were subjected to 30 or $45 \mathrm{~min}$ ischemia, followed by 120 min reperfusion (control). Anesthetic preconditioning was elicited with $2 \%$ sevoflurane for ten minutes prior to ischemia (SEVO-30, SEVO-45). Chloramphenicol (autophagy upregulator, $300 \mu \mathrm{M}$ ) was administered starting $20 \mathrm{~min}$ before ischemia and throughout reperfusion in SEVO-45 (SEVO-45+CAP). To inhibit autophagy, 3-methyladenine (3MA:10 $\mu \mathrm{M}$ ) was administered during sevoflurane administration in SEVO-45+CAP. Infarct size was determined by triphenyltetrazolium chloride stain. Tissue samples were obtained before ischemia to determine autophagy-related protein (microtubule-associated protein light chain I and II: LC3-I, II), Akt and glycogen synthase kinase $3 \beta$ (GSK3 $\beta$ ) expression using Western blot analysis. The effect of autophagy on calcium-induced mitochondrial permeability transition pore (MPTP) opening in isolated calcein-loaded mitochondria was assessed. Electron microscopy was used to detect autophagosomes.

Results: Infarct size was significantly reduced in SEVO-30, but not in SEVO-45. Chloramphenicol restored SPC lost by $45 \mathrm{~min}$ ischemia. There were more abundant autophagozomes and LC3-II expression was significantly increased in SEVO-45+CAP. Induction of autophagy before ischemia enhanced GSK3 $\beta$ phosphorylation and inhibition of calcium-induced MPTP opening. These effects were abolished by 3MA. 
Conclusions: Pre-ischemic induction of autophagy restores SPC lost by longer ischemic insult. This effect is associated with enhanced inhibition of MPTP by autophagy.

Key Words:

autophagy, chloramphenicol, ischemia-reperfusion, preconditioning, sevoflurane 


\section{Introduction}

Experimental $^{1-3}$ and clinical studies ${ }^{4,5}$ have demonstrated the use of volatile anesthetics as an additional therapeutic approach in the care of patients at risk of developing perioperative cardiac complications; known as volatile anesthetic preconditioning. ${ }^{6,7}$ The washout time of volatile anesthetics prior to ischemia ${ }^{8}$ and the duration of sustained ischemia ${ }^{9,10}$ have been shown to be crucial to successful volatile anesthetic cardioprotection. Kevin et al. demonstrated that sevoflurane cardioprotection is restricted to a range of ischemic durations of $25-40 \mathrm{~min}$ in isolated guinea pig hearts. ${ }^{9}$ Similarly, the neuroprotective effects of clinically relevant sevoflurane concentrations was observed only for ischemia of $\leq 30$ minutes. $^{10}$ Finding strategies that prolong cardioprotection by sevoflurane preconditioning during longer ischemic insults could be clinically useful.

Autophagy is a catabolic process through which damaged or long-lived protein and organelles are degraded using lysosomal degradative pathways. This is an evolutionally conserved process crucial for normal tissue homeostasis. Autophagy is considered one mode of cell death, termed "autophagic cell death". Certainly, necrosis and apoptosis contribute to cardiomyocyte death in ischemia-reperfusion injury. However, accumulating evidence suggests that autophagy is stimulated by ischemia and may contribute to cardiomyocyte survival. ${ }^{12,13}$ Yet, excessive self-digestion and degradation of essential cellular components by autophagy could be detrimental. ${ }^{14}$ To date, it is unclear whether upregulation of autophagy is protective or detrimental for ischemic myocardium.

Autophagy is triggered by opening of mitochondrial permeability transition pores (MPTP), which also play an important role in myocardial ischemia-reperfusion injury. ${ }^{15}$ Studies have shown that inactivation of glycogen synthase kinase $3 \beta$ (GSK3 $\beta$ ) by 
phosphorylation at $\mathrm{Ser}^{9}$ inhibits $\mathrm{MPTP}$ opening and protects cardiomyocytes against ischemia-reperfusion injury. ${ }^{16}$ It is not known whether pre-ischemic induction of autophagy affects MPTP. We hypothesized that pre-ischemic induction of autophagy restores loss of sevoflurane preconditioning seen with prolonged ischemic insult and this effect is associated with MPTP opening. Elucidating the role of autophagy in ischemia-reperfusion injury and finding strategies that prolong cardioprotection by sevoflurane preconditioning during longer ischemic insults could prove clinically useful in patients at cardiovascular risk during perioperative periods. 


\section{Methods}

This study was conducted in accordance with the Guidelines for Animal Research at Osaka Dental University with approval of the Animal Experiment Committee of Osaka Dental University, Osaka, Japan. These guidelines conform to the Guide for the Care and Use of Laboratory Animals from the National Academy of Science. Male Hartley guinea pigs were fed Lab Diet (RC4, Oriental Yeast, Tokyo, Japan) and water ad libitum. Chloramphenicol was purchased from Calbiochem (La Jolla, CA, USA) to induce autophagy. 3-methyladenine (3MA) was purchased from Sigma Aldrich (Ann Arbor, MI, USA) to inhibit autophagy.

\section{Isolated Heart Perfusion and Measurement of Function}

Guinea pigs (550-700g; 12-13 weeks old) received heparin (1000 units intraperitoneally), then were anesthetized with pentobarbital $\left(60 \mathrm{mg}^{-1} \cdot \mathrm{kg}^{-1}\right.$, intraperitoneally). Hearts were excised and arrested in cold iso-osmotic saline containing $20 \mathrm{mM} \mathrm{KCl}$. The aorta was cannulated and hearts were perfused at $70 \mathrm{mmHg}$ on a nonrecirculating isovolumic perfusion apparatus, using Krebs-Henseleit perfusate, and paced at 240 beats/min. ${ }^{3}$ Left ventricular developed pressure (LVDP) was measured using a 2.5 French, high-fidelity micromanometer (Nihon-Kohden, Tokyo, Japan) passed into a compliant latex balloon, inserted into the left ventricle, and recorded on a PowerLab 2/20 Data Recording System (ADInstruments, Hayward, Australia). The balloon was connected to a Y-adapter with one end used to advance the micromanometer and the other to fill the left ventricular balloon with bubble-free water to an end-diastolic pressure (LVEDP) of $10 \mathrm{mmHg}$. Coronary flow $(\mathrm{CF})\left(\mathrm{ml} \cdot \mathrm{min}^{-1} \cdot \mathrm{g}^{-1}\right)$ was measured by collecting effluent. Global ischemia 
was achieved by clamping the aortic inflow line. During ischemia, hearts were maintained at $37{ }^{\circ} \mathrm{C}$ by enclosure in a water-jacketed air chamber. Warmed perfusate in the lower part of the chamber saturated the air with humidity and prevented cooling by evaporation. Heart temperature was continuously monitored by a digital thermometer (PTW-100A, Unique Medical, Tokyo, Japan). Sevoflurane was insufflated passing the $95 \% \mathrm{O}_{2} / 5 \% \mathrm{CO}_{2}$ gas mixture through a calibrated vaporizer (ACOMA, Tokyo, Japan). Coronary perfusate samples were collected anaerobically from the aortic cannula for measurement of sevoflurane concentration by an organic vapor sensor (OSP, Saitama, Japan).

\section{Experimental Protocol}

Animals were assigned 10 groups ( $\mathrm{n}=8$ each; Figure 1). After 20 min equilibration, baseline LVDP, LVEDP and CF were recorded. Hearts were subjected to 30 min (control; CTL-30) or $45 \mathrm{~min}$ (control; CTL-45) ischemia followed by $120 \mathrm{~min}$ reperfusion. Anesthetic preconditioning was elicited by administration of sevoflurane (2\%) for $10 \mathrm{~min}$ followed by $10 \mathrm{~min}$ washout before $30 \mathrm{~min}$ or $45 \mathrm{~min}$ ischemia (SEVO-30, SEVO-45). For induction of autophagy, chloramphenicol $(300 \mu \mathrm{M})$ was administered starting 20 min before $45 \mathrm{~min}$ ischemia and throughout reperfusion in sevoflurane-treated (SEVO-45+CAP) or non-sevoflurane-treated animals (CAP-45) hearts. To inhibit autophagy, 3MA (10 $\mu \mathrm{M})$ was administered during sevoflurane administration for $20 \mathrm{~min}$ in SEVO-45+CAP (SEVO45+CAP+3MA) and its vehicle (saline) in CTL-45 (CTL-45+3MA) (Figure 1). Chloramphenicol was dissolved in distilled water and 3MA was dissolved in $0.04 \%$ ethanol, to final concentrations of $300 \mu \mathrm{M}$ and $10 \mu \mathrm{M}$, respectively. 


\section{Determination of Myocardial Infarct Size}

At experiment end, hearts were frozen at $-80{ }^{\circ} \mathrm{C}$ for $15 \mathrm{~min}$, then sliced into $2 \mathrm{~mm}$ thick transverse sections from apex to base (6 slices/heart). After defrosting, slices were incubated at $37{ }^{\circ} \mathrm{C}$ with $1 \%$ triphenyltetrazolium chloride (Sigma Chemicals, USA) photographed, and necrotic area was determined using Adobe Photoshop ${ }^{\circledR}$ CS (Adobe, San Jose, CA, USA) as previously described. ${ }^{3}$

\section{Western Blot Analysis}

Separate experiments were performed ( $\mathrm{n}=4$ per group) to examine expression of autophagy-related protein (microtubule-associated protein light chain I and II: LC3-I, II), Akt, GSK3 $\beta$ and cyclophilin D. Myocardial tissue samples were collected before ischemia, and homogenized in ice-cold homogenizing buffer as previously described. ${ }^{3}$ Homogenate was centrifuged at $1000 \mathrm{~g}$ for $5 \mathrm{~min}$ at $4{ }^{\circ} \mathrm{C}$. Supernatant was re-centrifuged at $10000 \mathrm{~g}$ for $15 \mathrm{~min}$ at $4{ }^{\circ} \mathrm{C}$ to obtain cytosolic fraction. The pellet was designated as crude mitochondrial fraction and was resuspended in homogenizing buffer with $1 \%$ TritonX-100, incubated on ice for $1 \mathrm{hr}$, then re-centrifuged at $10000 \mathrm{~g}$ for $15 \mathrm{~min}$ at $4{ }^{\circ} \mathrm{C}$. The resulting supernatant was used as mitochondrial fraction. Protein concentration was estimated with a Bradford assay. Equivalent amounts $(20 \mu \mathrm{g})$ of protein samples were loaded and separated on 10\% SDS-PAGE gradient gel, then electrically transferred overnight to a polyvinylidene difluoride membrane (Bio-Rad, Hercules, CA). After blocking with 5\% skim milk in Tris-buffered saline containing $0.1 \%$ Tween-20 (TBS-T), membranes were incubated for $2 \mathrm{~h}$ at $4{ }^{\circ} \mathrm{C}$ in TBS-T containing $5 \%$ skim milk and overnight with 1:200-1000 dilution of rabbit primary antibody for LC3 (Medical \& Biological Laboratories Co LTD, MA, USA), phospho Akt (Ser47), phospho GSK3 $\beta$ (Ser9) 
(Cell Signaling TECHNOLOGY, Boston, MA, USA) and cyclophilin D (Santa Cruz Biotechnology, Santa Cruz, CA, USA). Membranes were incubated with a 1:1000 dilution of horseradish peroxidase-labeled anti-rabbit immunoglobulin G (NA 934V, GE Healthcare, Buckinghamshire, United Kingdom). The same blot was stripped and re-blotted with $\alpha$-tubulin antibodies (Santa Cruz Biotechnology), total Akt and GSK3 $\beta$ (Cell Signaling TECHNOLOGY). Bound antibody signals were detected with enhanced chemiluminescence (Pierce Biotechnology, Rockford, IL, USA) and visualized using VersaDoc 5000 Imaging System (Bio-Rad, Hercules, CA). Quantitative analysis of band densities was performed with Quantity One software (Bio-Rad). Results are presented as the ratio of phospho Akt and phospho GSK3 $\beta$ to total (including non-phosphorylated and phosphorylated protein) Akt and GSK3 $\beta$, respectively.

\section{Studies in isolated cardiac mitochondria}

To investigate involvement of MPTP in cardioprotection by autophagy, mitochondria were isolated ( $n=4$ per group). After perfused and treated with sevoflurane, chloramphenicol and 3MA, isolated hearts were removed from the Langendorff apparatus, and homogenized in ice-cold MSTEB buffer as previously described. ${ }^{17}$ Extracted mitochondria were diluted in ice-cold respiratory buffer and incubated with $1 \mu \mathrm{M}$ calcein-AM (Invitrogen Molecular Probes, Carlsbad, USA) for $15 \mathrm{~min}$ at room temperature. Calcein-AM readily enters the mitochondria and is trapped in the matrix in its free form, which is fluorescent. After calcein was trapped in mitochondria, mitochondria were washed by $\mathrm{KCl}$ buffer. ${ }^{17}$ Calcein-loaded mitochondria were treated with 50 and $150 \mu \mathrm{M} \mathrm{Ca}^{++}$per milligram of protein, and were incubated for $10 \mathrm{~min}$ at room temperature. Mitochondrial fluorescences were acquired. Flow 
cytometric analysis was performed on FACS calibur ${ }^{\mathrm{TM}}$ (Becton Dickinson, Franklin lakes, NJ). Mitochondria labeled with calcein-AM were analyzed by flow cytometry in an instrument equipped with a $488 \mathrm{~nm}$ excitation source.

\section{Electron Microscopy}

To confirm induction of autophagy, cardiac specimens were obtained before sustained ischemia. They were fixed with phosphate-buffered $2.5 \%$ glutaraldehyde (pH 7.4) and were postfixed with $1 \%$ osmium tetroxide, after which they were conventionally prepared for transmission electron microscopy (H-800, Hitachi, Tokyo, Japan).

\section{Statistical Analysis}

Data are expressed as mean \pm SD. Statistical power analysis revealed a sample size of $\mathrm{n}=8$ would provide sufficient power $(0.8)$ to detect a difference between mean infarct size indices of $15 \%(\mathrm{SD}=9, \alpha=0.05)$. A group size of $\mathrm{n}=4$ was used for Western blot and calcein studies to provide a power of 0.8 to detect a difference between means of $20 \%$ ( $S D=10$, $\alpha=0.05)$. Hemodynamic data were tested for normal distribution and subsequently analyzed by a two-factor repeated-measures analysis of variance for time and treatment. If an overall difference between variables was observed, comparisons were performed as one-way ANOVA followed by Tukey's post-hoc test for inter-group differences and by Dunnett's for intra-group differences with baseline values as the reference time point. Analyses of infarct size, Western blot and mitochondrial calcein fluorescence were performed using one-way ANOVA followed by Student's t-test with Bonferroni's correction for multiple comparisons to avoid type I error. For changes within and between groups a two-tailed $\mathrm{p}$ value less than 0.05 was considered significant (SPSS17 for Windows, SPSS Japan, Tokyo, Japan). 


\section{Results}

Of 189 hearts, 7 were not used secondary to intractable ventricular fibrillation after reperfusion (3 in CTL-45, 2 in CAP-45, 1 in SEVO-45+3MA and 1 in SEVO$45+\mathrm{CAP}+3 \mathrm{MA})$ and one due to aortic rupture. Additional hearts were studied until each group had $n=8$ successful experiments for the infarct size study. The concentration of sevoflurane in the coronary perfusate after $10 \mathrm{~min}$ of exposure was $0.25 \pm 0.02 \mathrm{mM}$. Sevoflurane was not detected in the effluent during the baseline, ischemic, and reperfusion periods.

\section{Hemodynamics}

Baseline LVDP and CF were similar among the 10 groups. Sevoflurane, chloramphenicol or treatment with 3MA did not significantly affect LVDP or CF before ischemia. After 120 min reperfusion, only SEVO-30 had significantly higher LVDP and lower LVEDP compared to other groups. Treatment with 3MA alone did not affect the recovery of LVDP compared with CTL. There was no significant difference in CF among all the groups throughout experiments. This suggests that changes in CF could not account for improved contractile recovery in SEVO-30 (Table 1).

\section{Myocardial Infarct Size}

Myocardial infarct size in SEVO-30 was significantly reduced compared with control hearts (SEVO-30: $24 \pm 8 \%$ vs. CTL-30: $48 \pm 6 \%$, p<0.05). However, this effect was lost when the ischemic period was extended to $45 \min$ (SEVO-45: $53 \pm 6 \%$ vs. CTL-45: $54 \pm 11 \%$, $\mathrm{p}=\mathrm{NS}$ ). Chloramphenicol restored protection lost by prolonged ischemia in SEVO-45 
(SEVO-45+CAP: $34 \pm 8 \%$ vs. SEVO-45: $52 \pm 10 \%$, p<0.05). Restoration of cardioprotection by chloramphenicol was abolished by 3MA (SEVO-45+CAP+3MA: 51 $\pm 8 \%$ vs. CTL-45,

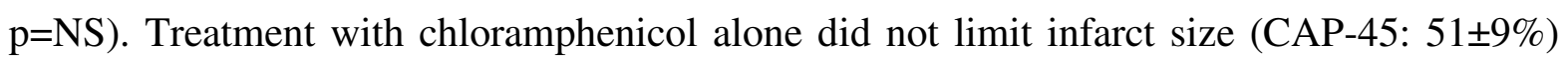
(Figure 2).

\section{Electron Microscopy}

Figure 3 shows representative electron microphotographs. Although autophagic vacuoles were rarely seen in cardiomyocytes of CTL (panel A), typical autophagosomes containing intracellular organelles, such as mitochondria and membrane-like structures (white arrows), were apparent after sevoflurane administration (panel B). Additional treatment with chloramphenicol (panel C) further increased the number of autophagosomes. Autophagosomes were diminished by addition of 3MA (panel D).

\section{Western Blot analysis}

In SEVO and CAP, LC3-II expression was increased compared with CTL. The combination of sevoflurane and chloramphenicol further increased this expression. These increased expressions of LC3-II were abolished by 3MA (Figure 4). The phosphorylation states of Akt and GSK3 $\beta$ after treatment with sevoflurane, cloramphenicol and 3MA are illustrated by a representative Western blot in Figure 5A. Total Akt and GSK3 $\beta$ were comparable in all samples. The ratio of phospho Akt to total Akt and phospho GSK3 $\beta$ to total GSK3 $\beta$ were significantly increased in SEVO and CAP compared with CTL (Figure 5B). This increase was not caused by unequal loading of the western blot, as shown by $\alpha$ tubulin detection. The combination of sevoflurane and chloramphenicol enhanced these 
increased expressions in SEVO-45+CAP. Administration of 3MA abolished these enhanced expressions in SEVO-45+CAP. There was no significant difference in expression of mitochondrial cyclophilin D between CTL and SEVO-45+CAP (Figure 5C).

Mitochondrial calcein fluorescence

The mitochondrial calcein fluorescence values after treatment with $\mathrm{Ca}^{2+}$ were taken as the values from which any reduction in fluorescence was measured. Exposure of mitochondria to $50 \mu \mathrm{M} \mathrm{Ca}^{2+}$ did not induce MPTP opening (Figure 6A). However, exposure of mitochondria to $150 \mu \mathrm{M} \mathrm{Ca}^{2+}$ induced MPTP opening, represented by a reduction in calcein fluorescence of $-66.9 \pm 13.0 \%$ in CTL. This $\mathrm{Ca}^{2+}$-induced reduction in calcein fluorescence was attenuated in the SEVO-45 and CAP-45 groups $(-40.0 \pm 8.0 \%,-44.0 \pm 1.8 \%$ vs. CTL-45, p $<0.05$, respectively; Figure $6 \mathrm{~B}$ ). This effect was enhanced by the SEVO45+CAP $(-15.5 \pm 0.3 \%$ vs. SEVO-45 and CAP-45, $\mathrm{p}<0.05)$, which was then abrogated by

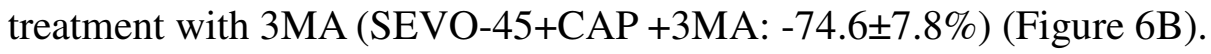




\section{Discussion}

This study demonstrated that induction of autophagy before ischemia restores the loss of sevoflurane preconditioning cardioprotection with prolonged ischemic insult. This effect is associated with enhanced phosphorylation of GSK3 $\beta$ before prolonged ischemia which results in elevation of the threshold of MPTP opening by $\mathrm{Ca}^{2+}$ overload, but not expression of mitochondrial cyclophilin D. There is a consensus that the use of volatile anesthetics during perioperative period could be a promising strategy to reduce ischemiareperfusion injury for patients at risk of developing cardiac complications. However, the beneficial effects of volatile anesthetics are limited to a certain range of sustained ischemia durations. Thus, strategies that prolong the protective effect of volatile anesthetics during longer ischemic insults should be explored. This study demonstrated for the first time that chloramphenicol treatment could be a novel strategy for this purpose.

Chloramphenicol, used as an antibiotic, is a cytochrome P450 monooxygenase inhibitor which inhibits mitochondrial protein synthesis, causing mitochondrial stress. Chloramphenicol induces autophagy and preconditioning-like cardioprotection, but the mechanisms underlying the cardioprotective effect remains to be established. Thus, we examined whether treatment with chloramphenicol affects expression of mitochondrial cyclophilin D, which is a component of MPTP that controls mitochondrial pore-dependent $\mathrm{Ca}^{+}$exchange, ${ }^{18,19}$ and is a critical mediator of cardioprotection by ischemic preconditioning. There were no significant differences in expression of mitochondrial cyclophilin D between CTL and SEVO-45+CAP. This suggests that cyclophilin D is not involved in cardioprotection by chloramphenicol. Chloramphenicol stimulates the autophagic transcript Atg 12 by inhibiting mitochondrial protein synthesis, which results in 
induction of autophagy. ${ }^{20}$ This study found that pretreatment with chloramphenicol before ischemia increased expression of LC3-II which leads to formation of autophagosomes. This was confirmed by electron microscopy.

McCormick et al. demonstrated that enhancing autophagy confers protection against ischemia-reperfusion injury in cardiomyocytes. ${ }^{21}$ Using in vivo swine models, Sala-Mercado et al. showed that induction of autophagy by chloramphenicol before ischemia limited infarct size. $^{22}$ However, the precise mechanisms by which pre-ischemic induction of autophagy confers cardioprotection remain to be elucidated. It has been demonstrated that induction of autophagy during ischemia is protective whereas further enhancement of autophagy is detrimental during reperfusion. ${ }^{23}$ Thus, it remains controversial whether autophagy is beneficial or harmful in ischemia-reperfusion injury.

MPTP opening is a crucial determinant of ischemia-reperfusion injury. ${ }^{24}$ MPTP opening has also been shown to induce autophagy. ${ }^{25}$ Elmore et al. demonstrated that autophagic stimulation caused an increase in spontaneously depolarizing mitochondria (presumably transient MPTP opening) in rat hepatocytes. ${ }^{26}$ Transient MPTP opening before prolonged ischemia makes the heart more tolerant to ischemia-reperfusion injury. ${ }^{27,28} \mathrm{We}$ previously demonstrated that heart exposure to sevoflurane increased the threshold of MPTP opening by $\mathrm{Ca}^{2+}$ loading. ${ }^{17}$ In the present study, exposure of control mitochondria to $150 \mu \mathrm{M}$ $\mathrm{Ca}^{2+}$ induced MPTP opening. This was attenuated in CAP and SEVO, and attenuated to an even greater degree in SEVO+CAP. These data suggests that transient MPTP opening by induction of autophagy with chloramphenicol increases the elevation of the threshold of MPTP opening by sevoflurane alone. 
The threshold for MPTP opening is increased by phosphorylation of GSK3 $\beta$ at $\operatorname{Ser}^{9}$ through phosphatidylinositol 3-kinase (PI3K)/Akt signaling. ${ }^{16}$ Whether there is an association between autophagy and GSK3 $\beta$ phosphorylation in reducing ischemiareperfusion injury is not known. Recently, it was reported that ultraviolet B-induced autophagy activated AMP-activated protein kinase, an important regulator of autophagy through inhibition/phosphorylation of GSK3 $\beta .{ }^{29}$ Our data demonstrated that induction of autophagy increased expression of phosphorylated GSK3 $\beta$ at $\operatorname{Ser}^{9}{ }^{9}$ accompanied by increased Akt phosphorylation. Both sevoflurane and chloramphenicol have been shown to activate PI3K/Akt signaling which is upstream of GSK3 $3 .{ }^{17,30}$ Class III PI3K is involved in the formation of autophagosomes, whereas class I PI3K inhibits the induction of autophagy via phosphorylation of Akt and the mammalian target of rapamycin (mTOR). ${ }^{31}$ Furthermore, there is evidence of self-regulation of autophagy by autophagy-induced inhibition of mTOR. ${ }^{32}$ Thus, the interaction of Akt with mTOR is multifaceted and bidirectional. Nevertheless, our data suggest that autophagy and PI3K-Akt-mTOR signaling might yield additive benefit against ischemia-reperfusion injury.

In contrast to the in vivo porcine study by Sala-Mercado et al, ${ }^{22}$ chloramphenicol alone did not limit infarct size in the present study, despite increased expression of autophagy-related protein LC3-II. However, chloramphenicol-induced autophagy did prolong sevoflurane preconditioning cardioprotection after a longer ischemic insult. Although the reason for the discrepancy remains to be elucidated, differences in ischemiareperfusion model and species could play a role.

The following study limitations should be acknowledged. First, GSK3 $\beta$ is a substrate of multiple pro-survival protein kinases. We cannot exclude ity that kinases other than 
PI3K/Akt signaling contributed to enhanced phosphorylation of GSK3 $\beta$. Second, the pharmacokinetics of 3MA are not well known, especially at high doses. The dose of 3MA used in this study $(10 \mu \mathrm{M})$ was low compared to previous studies $(5 \mathrm{mM}$ or $5 \mathrm{nM}) .^{21,29}$ However, this dose has been shown to effectively block LC3-II expression in H9c2 cells as was seen in this study. ${ }^{33}$

In conclusion, induction of autophagy by before ischemia restores the loss of sevoflurane preconditioning cardioprotection with prolonged ischemic insult. This effect is associated with enhanced phosphorylation of GSK3 $\beta$ before prolonged ischemia which is associated with enhanced inhibition of MPTP opening.

3317 words 


\section{Acknowledgments}

We thank Akiko Tsujimoto (Gifu University) for technical assistance.

\section{Declaration of interest}

The authors have no conflicts of interest to report.

\section{Funding}

This study was supported by Grant-in-Aid for Scientific Research (C) (grant number 23593008) from the Ministry of Education, Culture, Sports, Science and Technology of Japan (Masami Miyamae)(Tokyo, Japan). 


\section{References}

1. Kersten JR, Schmeling TJ, Pagel PS, Gross GJ, Warltier DC. Isoflurane mimics ischemic preconditioning via activation of K(ATP) channels: reduction of myocardial infarct size with an acute memory phase. Anesthesiology 1997; 87: 361-70

2. Kaneda K, Miyamae M, Sugioka S et al. Sevoflurane enhances ethanol-induced cardiac preconditioning through modulation of protein kinase $\mathrm{C}$, mitochondrial KATP channels, and nitric oxide synthase, in guinea pig hearts. Anesth Analg 2008; 106: 916.

3. Inamura $\mathrm{Y}$, Miyamae $\mathrm{M}$, Sugioka $\mathrm{S}$ et al. Aprotinin abolishes sevoflurane postconditioning by inhibiting nitric oxide production and phosphorylation of protein

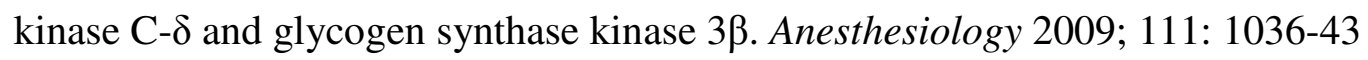

4. Garcia C, Julier K, Bestmann L et al. Preconditioning with sevoflurane decreases PECAM-1 expression and improves one-year cardiovascular outcome in coronary artery bypass graft surgery. Br J Anaesth 2005; 94: 159-65

5. Julier K, da Silva R, Garcia C et al. Preconditioning by sevoflurane decreases biochemical markers for myocardial and renal dysfunction in coronary artery bypass graft surgery: a double-blinded, placebo-controlled, multicenter study. Anesthesiology 2003; 98: 1315-27

6. De Hert SG, Turani F, Mathur S, Stowe DF. Cardioprotection with volatile anesthetics: mechanisms and clinical implications. Anesth Analg 2005; 100: 1584-93

7. De Hert SG. Anesthetic preconditioning: how important is it in today's cardiac anesthesia? J Cardiothorac Vasc Anesth 2006; 20: 473-6

8. Okusa C, Miyamae M, Sugioka S et al. Acute memory phase of sevoflurane preconditioning is associated with sustained translocation of protein kinase $\mathrm{C}-\alpha$ and $\varepsilon$, but not $\delta$, in isolated guinea pig hearts. Eur J Anaesthesiol 2009; 26: 582-8 
9. Kevin LG, Katz P, Camara AK et al. Anesthetic preconditioning: effects on latency to ischemic injury in isolated hearts. Anesthesiology 2003; 99: 385-91

10. Sigaut S, Jannier V, Rouelle D et al. The preconditioning effect of sevoflurane on the oxygen glucose-deprived hippocampal slice: the role of tyrosine kinases and duration of ischemia. Anesth Analg 2009; 108: 601-8

11. Aviv Y, Shaw J, Gang H, Kirshenbaum LA. Regulation of autophagy in the heart: "you only live twice". Antioxid Redox Signal 2011; 14: 2245-50

12. Yan L, Vatner DE, Kim SJ et al. Autophagy in chronically ischemic myocardium. Proc Natl Acad Sci U S A 2005; 102: 13807-12

13. Kanamori H, Takemura G, Goto $\mathrm{K}$ et al. Autophagy limits acute myocardial infarction induced by permanent coronary artery occlusion. Am J Physiol Heart Circ Physiol 2011; 300: H2261-71

14. Scarlatti F, Granata R, Meijer AJ, Codogno P. Does autophagy have a license to kill mammalian cells? Cell death and differentiation 2009; 16: 12-20

15. Halestrap AP, Clarke SJ, Javadov SA. Mitochondrial permeability transition pore opening during myocardial reperfusion-a target for cardioprotection. Cardiovascular research 2004; 61: 372-85

16. Juhaszova M, Zorov DB, Kim SH et al. Glycogen synthase kinase-3 $\beta$ mediates convergence of protection signaling to inhibit the mitochondrial permeability transition pore. The Journal of clinical investigation 2004; 113: 1535-49

17. Onishi A, Miyamae M, Kaneda K, Kotani J, Figueredo VM. Direct evidence for inhibition of mitochondrial permeability transition pore opening by sevoflurane preconditioning in cardiomyocytes: Comparison with cyclosporine A. European journal of pharmacology 2012; 675: 40-6 
18. Hausenloy DJ, Lim SY, Ong SG, Davidson SM, Yellon DM. Mitochondrial cyclophilin-D as a critical mediator of ischaemic preconditioning. Cardiovascular research 2010; 88: 67-74

19. Elrod JW, Wong R, Mishra S et al. Cyclophilin D controls mitochondrial poredependent $\mathrm{Ca}(2+)$ exchange, metabolic flexibility, and propensity for heart failure in mice. The Journal of clinical investigation 2010; 120: 3680-7

20 Prigione A, Cortopassi G. Mitochondrial DNA deletions and chloramphenicol treatment stimulate the autophagic transcript ATG12. Autophagy 2007; 3: 377-80

21. McCormick J, Suleman N, Scarabelli TM et al. STAT1 deficiency in the heart protects against myocardial infarction by enhancing autophagy. Journal of cellular and molecular medicine 2012; 16: 386-93

22. Sala-Mercado JA, Wider J, Undyala VV et al. Profound cardioprotection with chloramphenicol succinate in the swine model of myocardial ischemia-reperfusion injury. Circulation 2010; 122: S179-84

23. Matsui $\mathrm{Y}$, Kyoi S, Takagi $\mathrm{H}$ et al. Molecular mechanisms and physiological significance of autophagy during myocardial ischemia and reperfusion. Autophagy 2008; 4: 409-15

24. Hausenloy DJ, Ong SB, Yellon DM. The mitochondrial permeability transition pore as a target for preconditioning and postconditioning. Basic Res Cardiol 2009; 104: 189-202

25. Gustafsson $\AA$ B, Gottlieb RA. Autophagy in ischemic heart disease. Circ Res 2009; 104: $150-8$

26. Elmore SP, Qian T, Grissom SF, Lemasters JJ. The mitochondrial permeability transition initiates autophagy in rat hepatocytes. Faseb J 2001; 15: 2286-7 
27 Hausenloy D, Wynne A, Duchen M, Yellon D. Transient mitochondrial permeability transition pore opening mediates preconditioning-induced protection. Circulation 2004; 109: 1714-7

28. Saotome $\mathrm{M}$, Katoh $\mathrm{H}$, Yaguchi $\mathrm{Y}$ et al. Transient opening of mitochondrial permeability transition pore by reactive oxygen species protects myocardium from ischemia-reperfusion injury. Am J Physiol Heart Circ Physiol 2009; 296: H1125-32

29. Yang $\mathrm{Y}$, Wang $\mathrm{H}$, Wang $\mathrm{S}$ et al. GSK3 $\beta$ signaling is involved in ultraviolet Binduced activation of autophagy in epidermal cells. Int J Oncol 2012; 41: 1782-8

30. Li CH, Cheng YW, Liao PL, Yang YT, Kang JJ. Chloramphenicol causes mitochondrial stress, decreases ATP biosynthesis, induces matrix metalloproteinase13 expression, and solid-tumor cell invasion. Toxicol Sci 2010; 116: 140-50

31. Petiot A, Ogier-Denis E, Blommaart EF, Meijer AJ, Codogno P. Distinct classes of phosphatidylinositol 3'-kinases are involved in signaling pathways that control macroautophagy in HT-29 cells. The Journal of biological chemistry 2000; 275: $992-$ 8

32. He C, Klionsky DJ. Regulation mechanisms and signaling pathways of autophagy. Annu Rev Genet 2009; 43: 67-93

33. Cetrullo S, Tantini B, Flamigni F et al. Antiapoptotic and antiautophagic effects of eicosapentaenoic acid in cardiac myoblasts exposed to palmitic acid. Nutrients 2012; 4: $78-90$ 


\section{Figure Legends}

Figure 1: Schematic illustration of the experimental protocol used in this study. There are ten groups. After $20 \mathrm{~min}$ baseline period, isolated perfused hearts were subjected to 30 or $45 \mathrm{~min}$ global ischemia and 120min reperfusion. Anesthetic preconditioning was elicited by administration of $10 \mathrm{~min}$ of SEVO (2\%) with $10 \mathrm{~min}$ washout before 30 or $45 \mathrm{~min}$ ischemia. CAP and 3-MA was administered starting $20 \mathrm{~min}$ before $45 \mathrm{~min}$ ischemia and throughout the reperfusion period in hearts from SEVO-treated or non-SEVO-treated animals. Tissue samples were obtained before ischemia. CTL, control; CAP, Chloramphenicol; 3-MA, 3Methyladenine; SEVO, sevoflurane.

Figure 2: Infarct size as a percentage of LV in ten groups. After 120min reperfusion, infarct size was significantly reduced in SEVO-30 but not in SEVO-45 compared to corresponding CTL. Treatment with CAP in SEVO-45 restored the protection lost by prolonged ischemia in SEVO-45. CAP alone did not limit infarct size after $45 \mathrm{~min}$ of ischemia. Restoration of cardioprotection achieved by CAP was abolished by 3MA. 3MA alone did not affect infarct size. Data are presented as mean $\pm \mathrm{SD} ; * \mathrm{p}<0.05$ versus CTL-30; $\nmid \mathrm{p}<0.05$ versus SEVO-45. CTL, control; CAP, Chloramphenicol; 3-MA, 3-Methyladenine; SEVO, sevoflurane ( $\mathrm{n}=8$ for each group).

Figure 3: Electron microphotographs of cardiomyocytes, showing pre-ischemic induction of autophagosomes by reagents. Autophagosomes were rarely seen in the untreated control heart (panel A), were apparently observed (white arrows) after treatment with SEVO-45 (panel B). Autophagosomes appear to be more abundant after additional treatment with CAP 
(SEVO-45+CAP) compared with SEVO-45 alone (panel C). Appearance of autophagosomes strikingly diminished after treatment with 3MA (panel D). Nucl, nucleus. Bars, $1 \mu \mathrm{m}$.

Figure 4: Representative western blot analysis of LC3-I, II from left ventricular samples obtained before ischemia. The expression of LC3-II was slightly increased in SEVO and CAP compared to CTL. The ratio of LC3-II to LC3-I (LC3-II/LC3-I) was further increased in SEVO-45+CAP compared to SEVO-45 and CAP-45. Administration of 3-MA abolished this increased LC3-II/I in the SEVO-45+CAP group. ${ }^{*} \mathrm{p}<0.05$ versus CTL, ${ }^{\dagger} \mathrm{p}<0.05$ versus SEVO-45 and CAP-45; CAP, Chloramphenicol; LC3-I, II, microtubule-associated protein light chain I and II; 3-MA, 3-Methyladenine; SEVO, sevoflurane.

Figure 5: (A) Representative western blot of phosphorylated GSK3 $\beta$ from left ventricular samples acquired before ischemia. Expression of phosphorylated GSK3 $\beta$ was significantly increased in the SEVO-45 and CAP-45 group. The combination of SEVO and CAP further increased this expression of phosphorylated GSK3 $\beta$ and Akt in the SEVO-45+CAP group. Administration of 3MA abolished this enhanced expression of phosphorylated GSK3 $\beta$ in the SEVO-45+CAP group.

(B) Densitometric evaluation of four experiments as the $x$-fold increase in average light density vs. CTL. The results are presented as the ratio of the phosphorylation state to total protein. The average light intensity was multiplied by 100 to facilitate the presentation of an $\mathrm{x}$-fold increase. Data are presented as mean standard deviation. $* \mathrm{p}<0.05$ versus CTL-45, ${ }^{\dagger} \mathrm{p}<0.05$ versus SEVO-45, CAP-45 and SEVO-45+CAP+3MA 
(C) Cytosolic and mitochondrial expression of cyclophilin D in CTL, CAP-45 and SEVO45+CAP. There was no significant difference in expression between the three groups.

CTL, control; CAP, Chloramphenicol; CyF D, cyclophilin D; GSK3 $\beta$, glycogen synthase kinase-3; 3-MA, 3-methyladenine; PHB, prohibitin; SEVO, sevoflurane.

Figure 6: (A) Representative flow cytometric profile of isolated cardiac mitochondria loaded with calcein showing the effects of calcium $(50 \mu \mathrm{M})$ on mPTP opening as demonstrated by reductions in mitochondrial calcein fluorescence. Exposure of mitochondria to $50 \mu \mathrm{M} \mathrm{Ca}{ }^{2+}$ did not induce MPTP opening in any group. (B) Exposure of mitochondria to $150 \mu \mathrm{M} \mathrm{Ca}{ }^{2+}$ induced MPTP opening in CTL. This $\mathrm{Ca}^{2+}$-induced reduction in calcein fluorescence was attenuated in SEVO and CAP. This effect was enhanced by the combination of sevoflurane and chloramphenicol, which was then abrogated by treatment with 3MA. (C) Effect of calcium $(150 \mu \mathrm{M})$ on MPTP opening as demonstrated by reductions in mitochondrial calcein fluorescence. Data are mean \pm S.D. Percent change from control in the presence or absence of 3-MA $(10 \mu \mathrm{M}) .{ }^{*} \mathrm{p}<0.05$ versus CTL-45; ${ }^{\dagger} \mathrm{p}<0.05$ versus SEVO-45 and CAP-45 groups. CTL, control; CAP, Chloramphenicol; 3MA, 3-Methyladenine; SEVO, sevoflurane. $\mathrm{n}=4$ for each group

Figure 7: Schematic diagram of potential mechanisms of autophagy-induced cardioprotection conferred by chloramphenicol and sevoflurane. Phosphorylation of GSK3 $\beta$ by PI3-Akt pathway inhibits MPTP opening. Class III PI3K is involved in formation of autophagosomes whereas class I PI3K inhibits the induction of autophagy via phosphorylation of Akt and mTOR. Treatment with chloramphenicol or sevoflurane alone 
activates class III PI3K to induce autophagy. Simultaneous treatment with chloramphenicol and sevoflurane enhances this pathway which results in enhanced phosphorylation of GSK3 $\beta$ and elevation of the threshold of MPTP opening.

$\mathrm{PI} 3 \mathrm{~K}$ = phosphatidylinositol 3-kinase; Akt = protein kinase $\mathrm{B} ;$ GSK3 $\beta$ = glycogen synthase kinase $3 \beta ;$ mTOR $=$ mammalian target of rapamycin; $\mathrm{MPTP}=$ mitochondrial permeability transition pore. 
Table 1

TABLE 1. Hemodynamic Variables

\begin{tabular}{|c|c|c|c|c|c|c|c|c|c|c|c|c|}
\hline \multirow{3}{*}{ LVDP (mmHg) } & \multirow{2}{*}{\multicolumn{3}{|c|}{ Baseline }} & \multicolumn{9}{|c|}{ Reperfusion (min) } \\
\hline & & & & \multicolumn{3}{|c|}{30} & \multicolumn{3}{|c|}{60} & \multicolumn{3}{|c|}{120} \\
\hline & & & & & & & & & & & & \\
\hline CTL-30 & 109 & \pm & 11 & 39 & \pm & 8 & 32 & \pm & 12 & 26 & \pm & 9 \\
\hline SEVO-30 & 103 & \pm & 13 & 61 & \pm & $9 *$ & 58 & \pm & $8 *$ & 50 & \pm & $8 *$ \\
\hline CTL-45 & 108 & \pm & 13 & 21 & \pm & 6 & 25 & \pm & 13 & 24 & \pm & 9 \\
\hline SEVO-45 & 101 & \pm & 10 & 23 & \pm & 11 & 24 & \pm & 10 & 23 & \pm & 9 \\
\hline $\mathrm{CAP}+45$ & 109 & \pm & 11 & 21 & \pm & 7 & 21 & \pm & 6 & 21 & \pm & 6 \\
\hline SEVO-45+CAP & 101 & \pm & 14 & 21 & \pm & 5 & 22 & \pm & 5 & 21 & \pm & 4 \\
\hline CTL-45+3MA & 117 & \pm & 12 & 18 & \pm & 6 & 20 & \pm & 7 & 17 & \pm & 6 \\
\hline SEVO-30+3MA & 97 & \pm & 14 & 44 & \pm & $16^{\dagger}$ & 47 & \pm & 17 & 44 & \pm & 17 \\
\hline SEVO-45+3MA & 95 & \pm & 10 & 23 & \pm & 15 & 24 & \pm & 14 & 20 & \pm & 16 \\
\hline CAP-45+3MA & 105 & \pm & 15 & 25 & \pm & 11 & 23 & \pm & 10 & 20 & \pm & 10 \\
\hline CAP-45+SEVO+3MA & 97 & \pm & 11 & 19 & \pm & 9 & 26 & \pm & 16 & 26 & \pm & 16 \\
\hline \multicolumn{13}{|l|}{ LVEDP (mmHg) } \\
\hline CTL-30 & 10 & \pm & 0 & 47 & \pm & 14 & 51 & \pm & 18 & 55 & \pm & 15 \\
\hline SEVO-30 & 10 & \pm & 0 & 24 & \pm & $7 *$ & 24 & \pm & $5 *$ & 26 & \pm & $7 *$ \\
\hline CTL-45 & 10 & \pm & 0 & 67 & \pm & 7 & 57 & \pm & 13 & 53 & \pm & 14 \\
\hline SEVO-45 & 10 & \pm & 0 & 55 & \pm & 13 & 50 & \pm & 11 & 47 & \pm & 12 \\
\hline $\mathrm{CAP}+45$ & 10 & \pm & 0 & 53 & \pm & 10 & 48 & \pm & 10 & 45 & \pm & 10 \\
\hline SEVO-45+CAP & 10 & \pm & 0 & 53 & \pm & 6 & 51 & \pm & 10 & 49 & \pm & 11 \\
\hline CTL-45+3MA & 10 & \pm & 0 & 68 & \pm & 8 & 66 & \pm & 10 & 66 & \pm & 11 \\
\hline SEVO-30+3MA & 10 & \pm & 0 & 27 & \pm & $7 *$ & 25 & \pm & $9 *$ & 25 & \pm & $9 *$ \\
\hline SEVO-45+3MA & 10 & \pm & 0 & 72 & \pm & 19 & 62 & \pm & 17 & 60 & \pm & 19 \\
\hline CAP-45+3MA & 10 & \pm & 0 & 63 & \pm & 12 & 57 & \pm & 15 & 52 & \pm & 19 \\
\hline SEVO-45+CAP+3MA & 10 & \pm & 0 & 59 & \pm & 15 & 51 & \pm & 15 & 44 & \pm & 16 \\
\hline \multicolumn{13}{|l|}{ CF (mL) } \\
\hline CTL-30 & 28 & \pm & 6 & 21 & \pm & 5 & 21 & \pm & 5 & 20 & \pm & 8 \\
\hline SEVO-30 & 31 & \pm & 7 & 23 & \pm & 4 & 22 & \pm & 5 & 22 & \pm & 6 \\
\hline CTL-45 & 29 & \pm & 7 & 18 & \pm & 8 & 18 & \pm & 10 & 18 & \pm & 9 \\
\hline SEVO-45 & 28 & \pm & 10 & 19 & \pm & 11 & 17 & \pm & 11 & 17 & \pm & 11 \\
\hline $\mathrm{CAP}+45$ & 28 & \pm & 3 & 19 & \pm & 5 & 18 & \pm & 6 & 18 & \pm & 7 \\
\hline SEVO-45+CAP & 34 & \pm & 4 & 18 & \pm & 5 & 19 & \pm & 9 & 19 & \pm & 9 \\
\hline CTL-45+3MA & 37 & \pm & 6 & 20 & \pm & 8 & 18 & \pm & 8 & 18 & \pm & 8 \\
\hline SEVO-30+3MA & 29 & \pm & 8 & 18 & \pm & 7 & 19 & \pm & 11 & 20 & \pm & 11 \\
\hline SEVO-45+3MA & 27 & \pm & 5 & 13 & \pm & 5 & 13 & \pm & 5 & 11 & \pm & 5 \\
\hline CAP-45+3MA & 31 & \pm & 9 & 20 & \pm & 9 & 19 & \pm & 9 & 19 & \pm & 9 \\
\hline SEVO-45+CAP+3MA & 31 & \pm & 9 & 19 & \pm & 7 & 19 & \pm & 7 & 18 & \pm & 6 \\
\hline
\end{tabular}


Data are presented as mean \pm SD. LVDP=left ventricular developed pressure; LVEDP=left ventricular end-diastolic pressure; $\mathrm{CF}=$ coronary flow; $\mathrm{CTL}=$ control; $\mathrm{CAP}=$ chloramphenicol; $3 \mathrm{MA}=3$-Methyladenine; SEVO=sevoflurane; ${ }^{*} \mathrm{p}<0.05$ vs. CTL- $30,{ }^{\dagger} \mathrm{p}<0.05$ vs. SEVO-30 $\mathrm{n}=8$ for each group. 
Figure 1

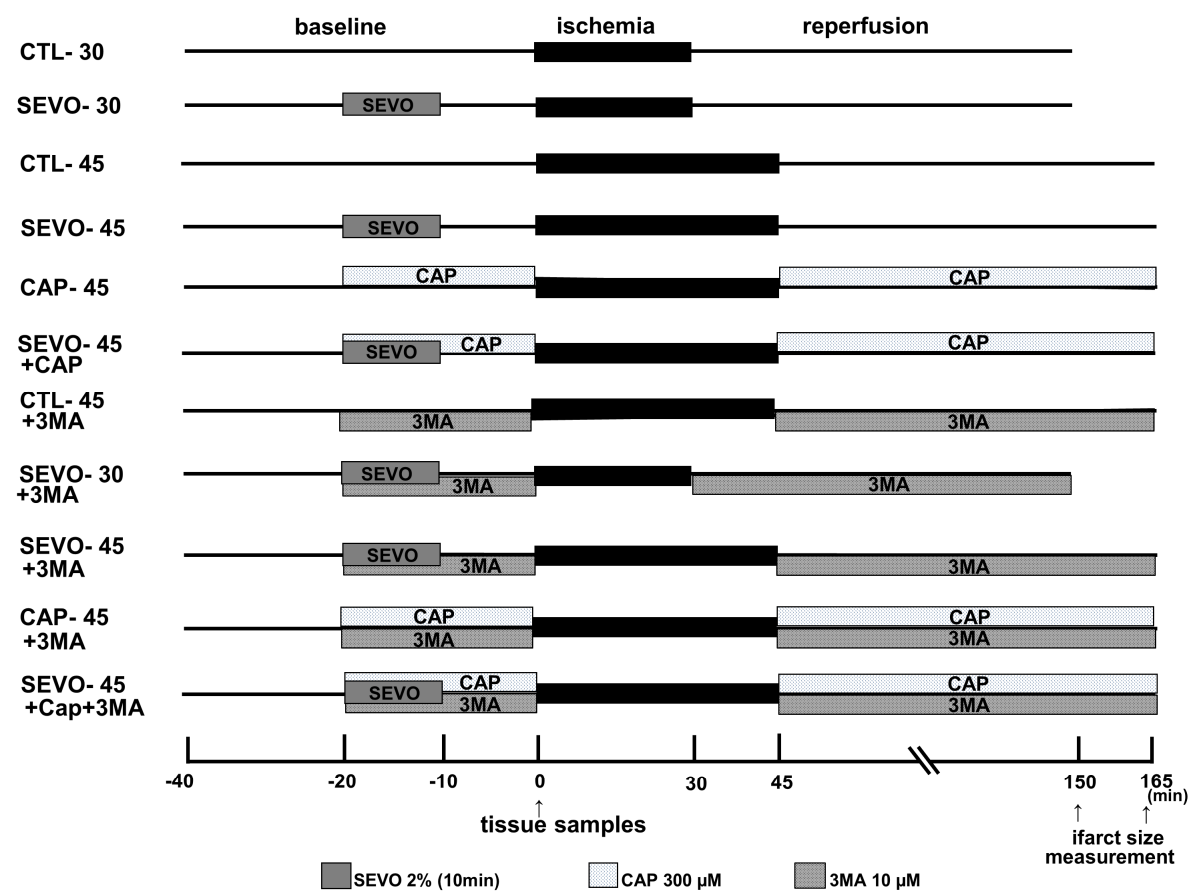


Figure 2

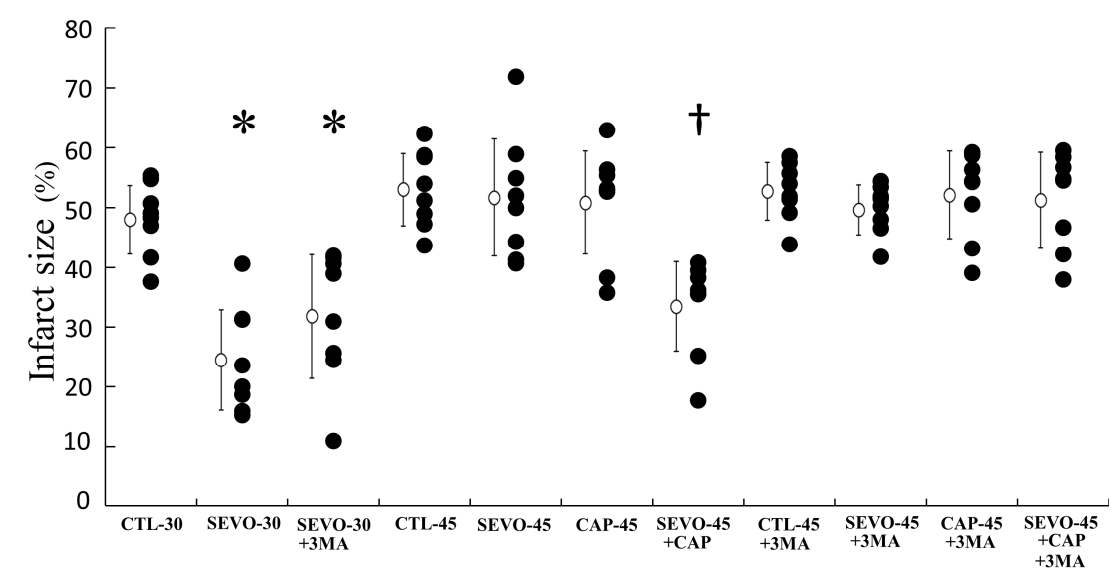


Figure 3
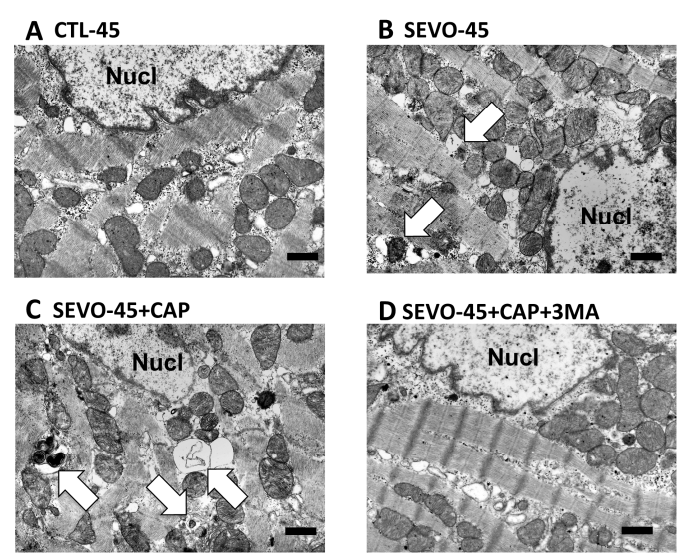

D SEVO-45+CAP+3MA

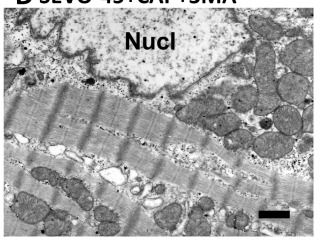


Figure 4

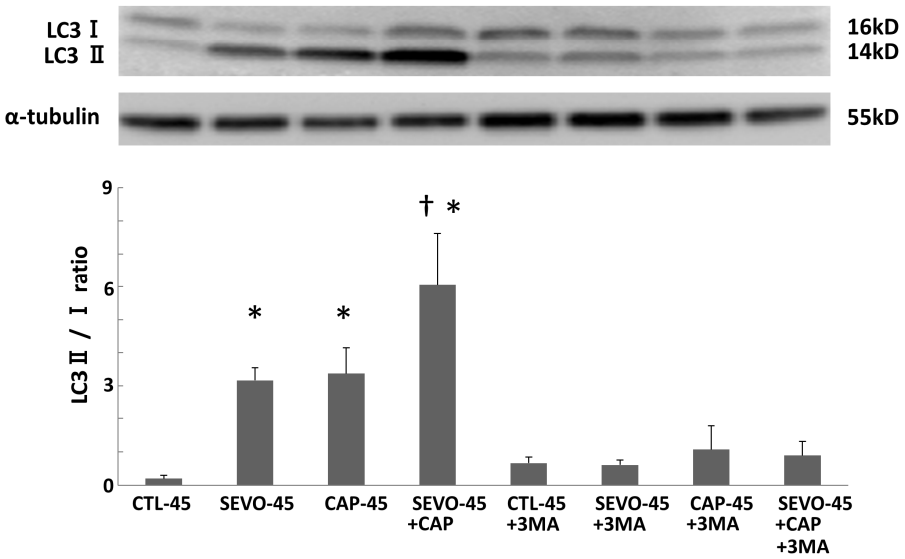


Figure 5

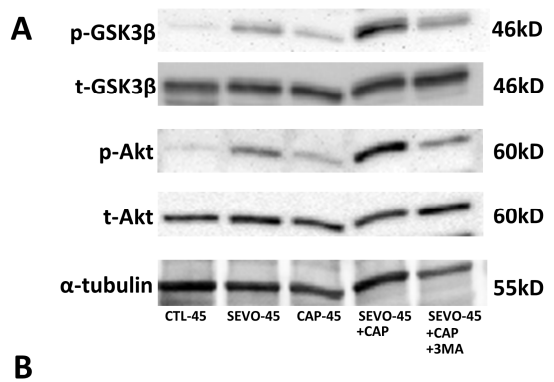

C
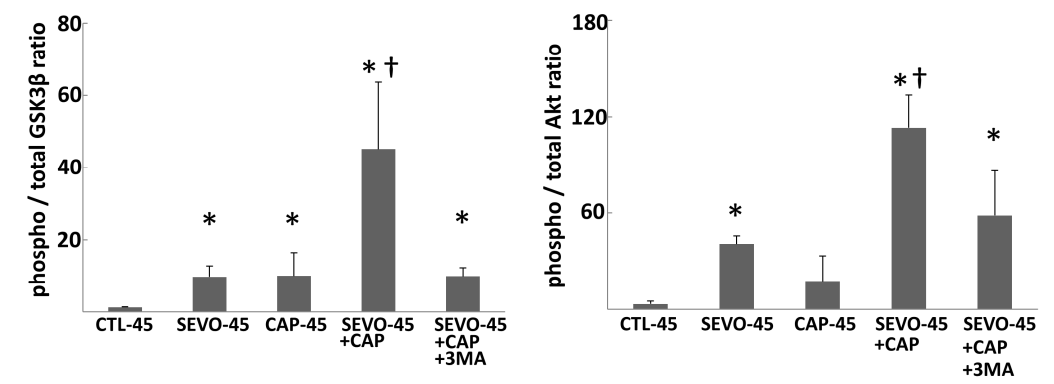
Figure 6
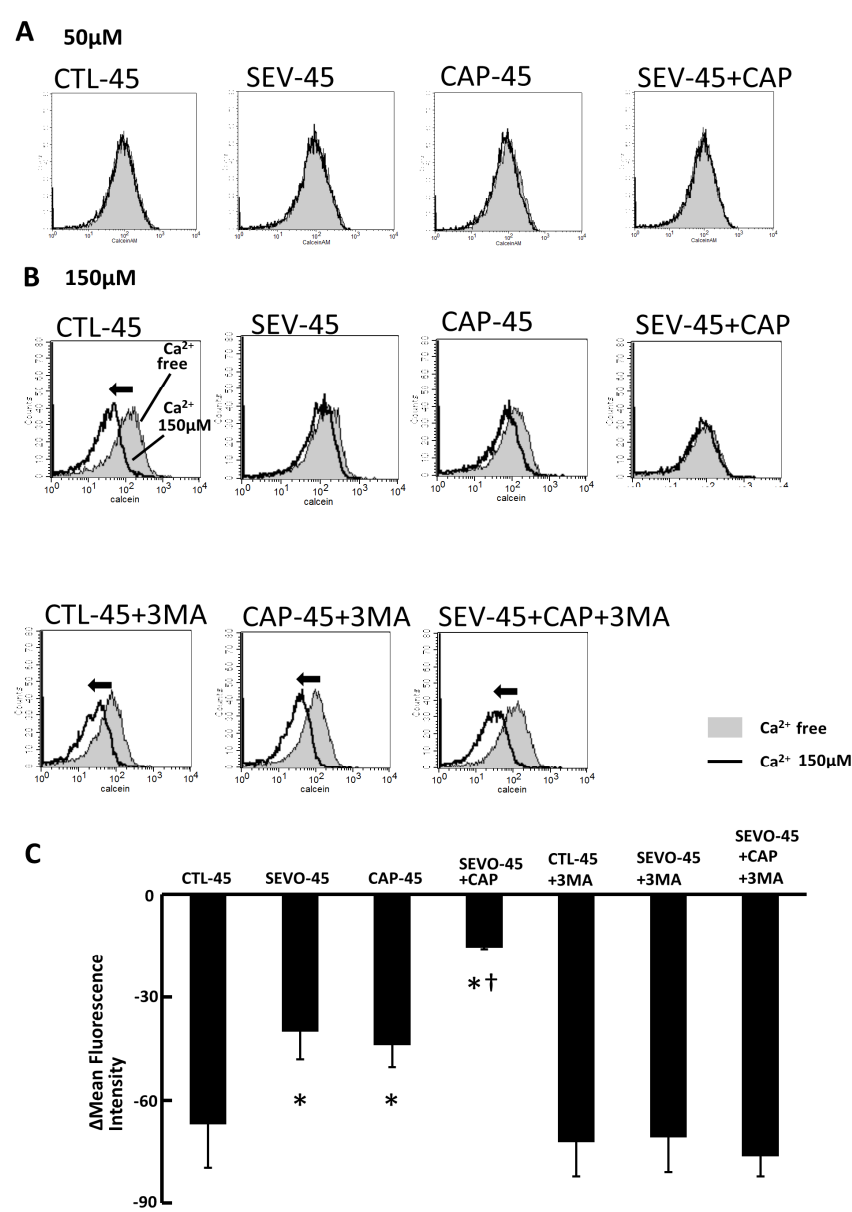
Figure 7

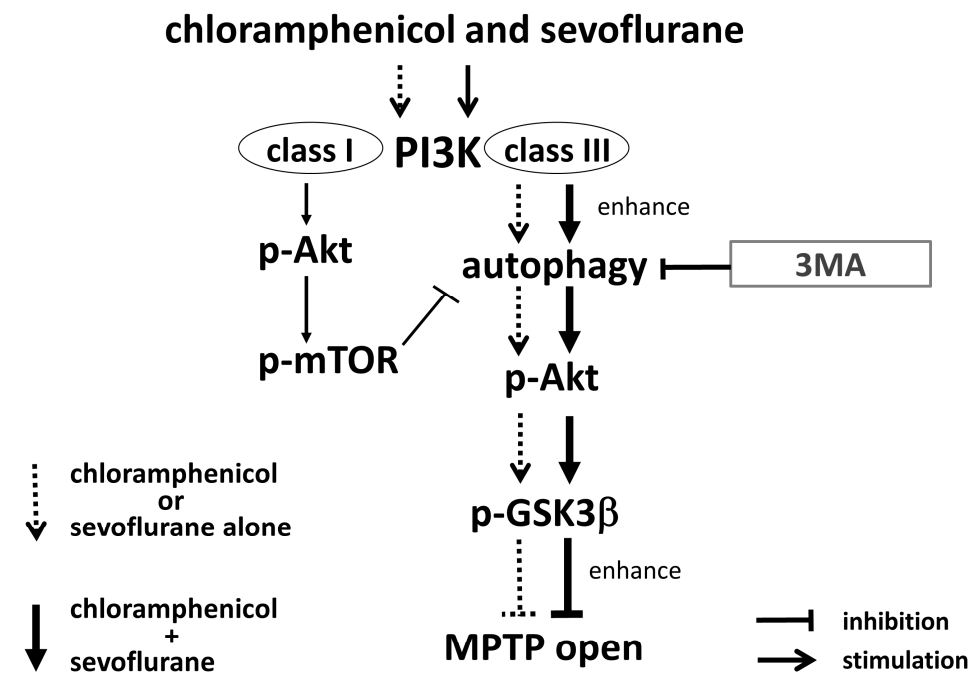

